\title{
High-level expression of divergent endodermal lineage markers in gonadal and extra-gonadal yolk sac tumors
}

\author{
Hadi Shojaei ${ }^{1}$, Hong Hong ${ }^{1}$ and Raymond W Redline ${ }^{2}$ \\ ${ }^{1}$ Pathology Resident, Department of Pathology, University Hospitals Case Medical Center, Case Western \\ Reserve University School of Medicine, Cleveland, OH, USA and ${ }^{2}$ Departments of Pathology and Reproductive \\ Biology, University Hospitals Case Medical Center and Case Western Reserve University School of Medicine, \\ Cleveland, $\mathrm{OH}, \mathrm{USA}$
}

\begin{abstract}
Yolk sac tumors occur at both gonadal and extra-gonadal sites. A recent case of ovarian endometrioid-pattern yolk sac tumor with strong diffuse expression of TTF-1 illustrated the potential for misdiagnosis due to divergent expression of endodermal lineage markers. The aim of this study was to investigate the expression of four divergent endodermal lineage markers, TTF-1, CDX2, Hep Par 1, and Napsin A, in gonadal and extra-gonadal yolk sac tumors of differing age, sex, and location (excluding foci of overt hepatoid differentiation). We identified 26 cases (5 ovarian, 15 testicular, and 6 extra-gonadal) containing yolk sac tumor as identified by typical histology and confirmed by positive immunohistochemical staining for alpha-fetoprotein and glypican-3. Mixed or ambiguous foci were confirmed by immunohistochemistry (SALL4 positive and Oct-4 negative). The relative proportion of three histologic patterns: reticular/cystic, solid/myxoid, and glandular was estimated. Percent positivity for the four divergent endodermal lineage markers was compared within yolk sac tumor areas according to site, age group, and histologic pattern. High-level $(>25 \%)$ staining for one or more divergent endodermal lineage markers was seen in eleven cases: Hep Par 1 in seven cases, all post-pubertal, TTF-1 in four cases, two ovarian and two extra-gonadal, and CDX2 in three cases, with no age or site predilection. No case highly expressed all three divergent endodermal lineage markers, but four co-expressed high levels of two markers: two ovarian yolk sac tumors with TTF-1 and Hep Par 1, one testicular yolk sac tumor with CDX2 and Hep Par 1, and one extra-gonadal yolk sac tumors with TTF-1 and CDX2. While no absolute correlation of high-level divergent endodermal lineage marker expression with histologic subtype was observed, TTF-1 and CDX2 expression was predominantly seen in reticular/cystic and glandular areas while Hep Par 1 was most frequent in myxoid/solid and glandular areas.

Modern Pathology (2016) 29, 1278-1288; doi:10.1038/modpathol.2016.131; published online 22 July 2016
\end{abstract}

Yolk sac tumors are malignant germ cell tumors of primitive endodermal differentiation. They can arise as pure tumors or mixed with other germ cellderived elements in male or female gonads or at extra-gonadal sites, generally along the midline (eg, mediastinum, retroperitoneum, and sacrum). ${ }^{1-5}$ Yolk sac tumor is the third most common form of germ cell tumor in the ovary (after teratoma and

Correspondence: Professor RW Redline, MD, Pathology and Reproductive Biology, Department of Pathology, University Hospitals Case Medical Center, Case Western Reserve University School of Medicine, 11100 Euclid Avenue, Cleveland, OH 44106, USA.

E-mail: raymondw.redline@UHhospitals.org

Received 28 March 2016; revised 2 June 2016; accepted 3 June 2016; published online 22 July 2016 dysgerminoma) and is a common component of mixed malignant germ cell tumors of the adult testis. Most ovarian yolk sac tumors occur as pure neoplasms with no age predilection (pre-pubertal vs post-pubertal), whereas pure testicular yolk sac tumor is rare in adults but the most common malignant testicular germ cell tumor in children $\leq 2$ years of age ${ }^{6-9}$ Extra-gonadal yolk sac tumors often arise as small foci in a background of immature teratoma, most commonly in children in the sacrococcygeal region, but can occur at older ages in many locations with mediastinum and pituitary representing other frequent sites.

Correct identification of yolk sac tumor or a yolk sac tumor component within other germ cell tumors is extremely important because it usually augurs an aggressive clinical course, requires distinct 
chemotherapeutic regimens, and is associated with expression of alpha-fetoprotein, which may be used as a circulating tumor marker to monitor therapy and detect recurrence. However, yolk sac tumor elements can be very small and demonstrate a wide variety of histological patterns that can be overlooked in teratomas or mixed malignant germ cell tumors.

A second issue, arising from their primitive endodermal differentiation, is that yolk sac tumors can express a number of antigens more commonly associated with tumors arising in differentiated organs of endodermal origin (eg, lung, thyroid, and gastrointestinal tract). ${ }^{10-15}$ Such divergent endodermal lineage marker expression can potentially lead to misinterpretation or delays in diagnosis, especially with small biopsy specimens. In this study, we undertook a systematic evaluation of the frequency, extent, and patterns of expression of four divergent endodermal lineage markers (TTF-1, CDX2, Hep Par 1, and Napsin A), in gonadal and extragonadal yolk sac tumors of both children and adults.

\section{Materials and methods}

\section{Study Design and Patient Selection}

Cases accessioned between 1999 and 2013 with a diagnosis of yolk sac tumor were retrieved from the surgical pathology files at University Hospitals Case Medical Center. Only patients with surgery at this institution were included (no outside consults) and complete slides and blocks were available for all cases. Hematoxylin and eosin-stained slides and previously performed immunohistochemical stains were reviewed to confirm the pathologic diagnosis of yolk sac tumor. In some cases, immunohistochemical stains were repeated due to poor technical quality in the originals. All slides were separately evaluated by two pathologists (RR and $\mathrm{HH}$ ). In cases of disagreement, consensus was achieved by reviewing slides together at a dual headed microscope. A total of 26 yolk sac tumors had archival formalinfixed, paraffin-embedded tissue blocks available for further study: five ovarian, fifteen testicular, and six extra-gonadal. Six cases were from young children (pre-pubertal, $<12$ years old) and twenty from teenagers or adults. Study approval was obtained from the University Hospitals Case Medical Center Institutional Review Board.

\section{Immunohistochemistry}

All cases were evaluated for the following immunohistochemical markers: octomer-binding factor 4 (Oct-4), spalt-like transcription factor 4 (SALL4), alpha-fetoprotein (AFP), glypican-3 (Glyp-3), thyroid transcription factor-1 (TTF-1), caudal type homeobox 2 (CDX2), hepatocyte paraffin 1 (Hep Par 1), and Napsin A. All staining was performed by the Immunohistochemistry Laboratory of the University
Hospitals Case Medical Center. Briefly, unstained $4 \mu \mathrm{m}$ sections on whole charged slides were prepared from paraffin blocks and baked for $30 \mathrm{~min}$ at $60{ }^{\circ} \mathrm{C}$ in a Boekel Lab oven. For AFP (Clone C3, Leica Biosyolk sac tumorems, Bannockburn, IL), TTF-1 (Clone SPT24, Leica), SALL4 (clone 6E3, Cell Marque, Rocklin, CA), Napsin A (Rabbit polyclonal, Cell Marque) and Hep Par 1 (Clone OCH1E5, Cell Marque), the slides were processed using a Bondmax (Leica) automated immunostainer. These slides were deparaffinized and antigen retrieved with Bond Epitope Retrieval Solution 1 (Leica) and a citratebased solution (pH 6.0) at $100^{\circ} \mathrm{C}$ for TTF-1 (20 min) and Hep Par 1 (15 min). Antigen retrieval for SALL4 and Napsin A was done with Bond Epitope Retrieval Solution 2 (Leica) and an EDTA-based solution (pH 9.0) at $100^{\circ} \mathrm{C}$, for $20 \mathrm{~min}$. For AFP, antigen retrieval was not performed. All primary antibodies were incubated for $15 \mathrm{~min}$ at room temperature and subsequently counterstained onboard with the automated instruments. The Bond Polymer Refine Detection (Leica) was used for these assays. For CDX2 (Clone EPR2764Y, Cell Marque) and Glyp-3 (Clone GC33, Ventana Medical Syolk sac tumorems, Tucson, AZ), slides were processed using a Bench Mark Ultra Automated Immunostainer (Ventana). These slides were deparaffinized, antigen retrieved for 36 and 64 min respectively for Glyp-3 and CDX2 with Cell Conditioning 1 (Ventana), a Tris-based buffer with a slightly basic $\mathrm{pH}$ solution at $95^{\circ} \mathrm{C}$. All primary antibodies were incubated for $15 \mathrm{~min}$ at $37^{\circ} \mathrm{C}$ and subsequently counterstained onboard with the automated instruments. The ultra View Universal DAB Detection Kit (Ventana) was used for these assays.

Yolk sac tumor areas were defined by histology and confirmed by expression of AFP and Glyp-3. In problematic cases, yolk sac tumor was distinguished from other germ cell tumor types by positive staining for SALL4 and negative staining for Oct-4. Relative percentages within the yolk sac tumor component of three major histologic subtypes were assessed: reticular/cystic (including parietal, endodermal sinus, papillary, and labyrinthine variants), solid/ myxoid, and glandular (enteric, endometrioid, or alveolar subtypes). Percent positivity for the primitive endodermal markers AFP and Glyp-3 and the mature endodermal markers TTF-1, CDX2, Hep Par 1, and Napsin A was compared within yolk sac tumor areas according to site, age group, and histologic pattern. Three groups were distinguished based on percentage positivity of tumor cells: absent to $<5 \%$ positive, $5-24 \%$ positive, and $\geq 25 \%$ positive. Percentage positivity of $25 \%$ or more was arbitrarily considered as high-level positive staining.

\section{Results}

The clinical and pathologic features of our 26 cases of yolk sac tumor are summarized in Table 1 . 
Table 1 Clinicopathologic data of yolk sac tumor cases

\begin{tabular}{|c|c|c|c|c|c|c|c|c|c|c|c|c|c|c|c|}
\hline & \multirow[b]{2}{*}{$\begin{array}{c}\text { Case } \\
\text { number }\end{array}$} & \multirow[b]{2}{*}{ Site } & \multirow[b]{2}{*}{$\begin{array}{l}\text { Primary vs } \\
\text { metastasis }\end{array}$} & \multirow[b]{2}{*}{ Sex/Age } & \multirow[b]{2}{*}{$\begin{array}{l}\text { Germ cell tumor } \\
\text { components }\end{array}$} & \multicolumn{3}{|c|}{ Histologic patterns } & \multicolumn{3}{|c|}{ YST panel } & \multicolumn{4}{|c|}{ ELM panel } \\
\hline & & & & & & $\begin{array}{l}\text { Reticular/ } \\
\text { Microcystic }\end{array}$ & $\begin{array}{l}\text { Myxoid/ } \\
\text { Solid }\end{array}$ & Glandular & SALL4 & $A F P$ & Glyp-3 & $T T F-1$ & $C D X 2$ & Hep Par 1 & Napsin $A$ \\
\hline \multirow{20}{*}{ Gonadal } & 1 & OV & $\mathrm{P}$ & $\mathrm{F} / 13 \mathrm{Y}$ & YS, DG & 75 & 20 & 5 & 100 & 60 & 20 & 0 & 30 & 7 & 0 \\
\hline & 2 & OV & $\mathrm{P}$ & $\mathrm{F} / 18 \mathrm{Y}$ & YS & 90 & 10 & 0 & 100 & 7 & 60 & 40 & 2 & 60 & 0 \\
\hline & 3 & OV & $\mathrm{P}$ & $\mathrm{F} / 19 \mathrm{Y}$ & YS, T, DG & 90 & 10 & 0 & 100 & 20 & 15 & 3 & $1 \overline{5}$ & 1 & 0 \\
\hline & 4 & OV & $\mathrm{P}$ & $\mathrm{F} / 25 \mathrm{Y}$ & YS & 90 & 10 & - & 100 & 10 & 50 & 1 & 5 & 10 & 0 \\
\hline & 5 & OV & $\mathrm{P}$ & $\mathrm{F} / 31 \mathrm{Y}$ & YS & 5 & 10 & 85 & 100 & 5 & 20 & 70 & 7 & 90 & 0 \\
\hline & 6 & TS & $\mathrm{P}$ & $\mathrm{M} / 3 \mathrm{M}$ & YS, T & 90 & 0 & 10 & 100 & 20 & 100 & 5 & 5 & 5 & 0 \\
\hline & 7 & TS & $\mathrm{P}$ & $\mathrm{M} / 16 \mathrm{Y}$ & YS, T, S & 20 & 5 & 75 & 100 & 7 & 20 & 5 & 5 & 5 & 3 \\
\hline & 8 & TS & $\mathrm{P}$ & $\mathrm{M} / 17 \mathrm{Y}$ & YS, T, S, EC & 100 & 0 & 0 & 100 & 50 & 1 & 0 & 1 & 1 & 0 \\
\hline & 9 & TS & $\mathrm{P}$ & $\mathrm{M} / 17 \mathrm{Y}$ & YS, T, EC, CH & 50 & 30 & 20 & 100 & 7 & 7 & 10 & 15 & 2 & 0 \\
\hline & 10 & $\mathrm{~T}$ & $\mathrm{P}$ & $\mathrm{M} / 20 \mathrm{Y}$ & YS, T, S, CH & 40 & 30 & 30 & 100 & 2 & 10 & 1 & 10 & 30 & 0 \\
\hline & 11 & TS & $\mathrm{P}$ & $\mathrm{M} / 21 \mathrm{Y}$ & YS, T, EC, CH & 45 & 50 & 5 & 100 & 1 & 25 & 0 & 1 & 60 & 0 \\
\hline & 12 & TS & $\mathrm{P}$ & $\mathrm{M} / 28 \mathrm{Y}$ & YS, T, S, EC & 50 & 25 & 25 & 100 & 1 & 15 & 0 & 20 & 0 & 0 \\
\hline & 13 & TS & $\mathrm{P}$ & $\mathrm{M} / 31 \mathrm{Y}$ & YS, S, EC, CH & 10 & 0 & 90 & 100 & 10 & 40 & 0 & 20 & 60 & 0 \\
\hline & 14 & TS & $\mathrm{P}$ & $\mathrm{M} / 33 \mathrm{Y}$ & YS, S, EC & 90 & 5 & 5 & 100 & 10 & 60 & 2 & 2 & 3 & 0 \\
\hline & 15 & LN & $\mathrm{M}$ & $\mathrm{M} / 33 \mathrm{Y}$ & $\mathrm{Y}, \mathrm{EC}$ & 0 & 100 & 0 & 100 & 10 & 5 & 0 & 5 & 20 & 0 \\
\hline & 16 & TS & $\mathrm{P}$ & $\mathrm{M} / 35 \mathrm{Y}$ & YS, S, EC & 90 & 10 & 0 & 100 & 2 & 60 & 0 & 1 & 40 & 0 \\
\hline & 17 & LN & $\mathrm{M}$ & $\mathrm{M} / 36 \mathrm{Y}$ & YS, T & 30 & 30 & 40 & 100 & 100 & 15 & 0 & 80 & 80 & 0 \\
\hline & 18 & $\mathrm{TS}$ & $\mathrm{P}$ & $\mathrm{M} / 37 \mathrm{Y}$ & YS, T & 85 & 10 & 5 & 100 & 80 & 50 & 0 & 7 & 2 & 0 \\
\hline & 19 & TS & $\mathrm{P}$ & $\mathrm{M} / 42 \mathrm{Y}$ & YS, S, EC & 40 & 60 & 0 & 100 & 7 & 70 & 15 & 5 & 1 & 0 \\
\hline & 20 & TS & $\mathrm{P}$ & $\mathrm{M} / 42 \mathrm{Y}$ & YS, T, EC & 100 & 0 & 0 & 100 & 40 & 10 & 5 & 2 & 5 & 0 \\
\hline \multirow[t]{6}{*}{ Extra-gonadal } & 21 & SC & $\mathrm{P}$ & $\mathrm{F} / 5 \mathrm{D}$ & YS, T & 75 & 25 & 0 & 100 & 50 & 50 & 0 & 1 & 0 & 0 \\
\hline & 22 & $\mathrm{~N}$ & $\mathrm{P}$ & $\mathrm{M} / 9 \mathrm{MO}$ & YS & 80 & 20 & 0 & 100 & 90 & 50 & 25 & 1 & 5 & 0 \\
\hline & 23 & SC & $\mathrm{P}$ & $\mathrm{F} / 14 \mathrm{MO}$ & YS & 100 & 0 & 0 & 100 & 20 & 30 & 1 & 1 & 0 & 0 \\
\hline & 24 & SC & $\mathrm{P}$ & $\mathrm{F} / 2 \mathrm{Y}$ & YS & 90 & 10 & 0 & 100 & 70 & 30 & 60 & 40 & 5 & 0 \\
\hline & 25 & SC & $\mathrm{P}$ & $\mathrm{M} / 2 \mathrm{Y}$ & YS & 90 & 5 & 5 & 100 & 10 & 15 & 1 & 2 & 1 & 0 \\
\hline & 26 & LN & $\mathrm{M}$ & $\mathrm{M} / 27 \mathrm{Y}$ & YS & 90 & 10 & 0 & 100 & 7 & 10 & 5 & 0 & 50 & 0 \\
\hline
\end{tabular}

Abbreviations: CH, choriocarcinoma; D, day; DG, dysgerminoma; EC, embryonal carcinoma; ELM, endodermal lineage markers; EST, endodermal sinus tumor; F, female; LN, lymph node; M, male; MGCT, malignant germ cell tumor; MO, month; MT, mature; N, nasal cavity; OV, ovary; S, seminoma; SC, sacrococcygeal; T, teratoma; TS, testis; Y, year; YS, yolk sac tumor; YST, yolk sac tumor. 
Table 2 Number of cases and level of divergent endodermal lineage marker staining for gonadal and extra-gonadal YST

\begin{tabular}{|c|c|c|c|c|}
\hline Primary site & Divergent ELM & Negative to low ( $\leq 5 \%$ positive) & Intermediate (5-24\% positive) & High ( $\geq 25 \%$ positive) \\
\hline \multirow[t]{3}{*}{ Ovary $N=5$} & TTF-1 & 3 & 0 & 2 \\
\hline & $\mathrm{CDX} 2$ & 2 & 2 & 1 \\
\hline & Hep Par 1 & 1 & 2 & 2 \\
\hline \multirow{3}{*}{ Testis $N=15$} & TTF-1 & 13 & 2 & 0 \\
\hline & CDX2 & 9 & 5 & 1 \\
\hline & Hep Par 1 & 9 & 2 & 4 \\
\hline \multirow[t]{3}{*}{ Extra-gonadal $N=6$} & TTF-1 & 4 & 0 & 2 \\
\hline & CDX2 & 5 & 0 & 1 \\
\hline & Hep Par 1 & 5 & 0 & 1 \\
\hline
\end{tabular}

Patients ranged in age from 3 months to 42 years at diagnosis. Cases were separately analyzed by site (5 ovarian, 15 testicular, and 6 extra-gonadal) and age (6 pre-pubertal and 20 post-pubertal). Three cases were lymph-node metastases; two derived from known testicular primaries, the other a supraclavicular lymph node from an unknown primary tumor. The remainder were primary tumors. All ovarian cases were post-pubertal. One of 15 testicular cases was pre-pubertal. Five of the six extragonadal yolk sac tumors were pre-pubertal; four arising in the sacrococcygeal region and the other in the nasal cavity. All testicular yolk sac tumors arose within mixed malignant germ cell tumors, while 8 of the 11 tumors from other sites were pure yolk sac tumors. The remaining three mixed cases were in a background of immature teratoma (two adult ovarian and one pre-pubertal extra-gonadal).

Microscopically, 16 cases showed a predominant $(\geq 75 \%)$ reticular/microcyolk sac tumoric pattern. Three of the remaining cases were predominantly glandular and three had a substantial $(\geq 50 \%)$ solid/ cystic component. The other three cases were more heterogeneous. Extra-gonadal cases were predominantly reticular/cystic with lesser components of myxoid/solid pattern and none showed more than a focal glandular pattern. A predominant glandular pattern was seen in three cases, one ovarian (endometrioid-type) and two testicular (enteric-types). No areas of alveolar type glandular, polyvesicular vitelline, or overt hepatoid differentiation were identified. For the purposes of this study, the term reticular/cystic encompassed all of the minor variants sometimes grouped under the heading 'classic pattern' (see Materials and methods).

All yolk sac tumors expressed SALL4, AFP, and Glyp-3, SALL4 diffusely staining all cells. Ambiguous foci and the extent of yolk sac tumor in mixed malignant germ cell tumors were defined by positivity for SALL4 and negativity for Oct-4. This differential staining profile correlated well with histologic features in the more classical areas of yolk sac tumor and was useful in distinguishing yolk sac tumors from embryonal carcinoma, choriocarcinoma, and immature teratoma, particularly in mixed malignant germ cell tumors of the testis. Mean percent positivity for AFP was highest in prepubertal yolk sac tumors (43 vs $18 \%$ ), but could be very focal ( $<5 \%$ in five cases). Glyp-3 was more consistently expressed with a mean percent positivity of around $30 \%$ at all ages and in all locations.

Four antigens reflecting possible divergent endodermal lineage markers expression, TTF-1, CDX2, Hep Par 1, and Napsin A, were assessed by immunohistochemical staining in each case. Only one case showed positive staining for Napsin A (a postpubertal testicular tumor with weak staining in $3 \%$ of cells) and this marker will not be further discussed. The percentage of yolk sac tumor cells staining positively for each endodermal lineage marker in individual cases is shown in Table 1; and the proportion of cases showing absent to low ( $\leq 5 \%$ positivity), intermediate $(6-24 \%$ positivity), and high ( $\geq 25 \%$ positivity) level expression for each marker by site of origin is summarized in Table 2 . High-level expression for at least one divergent endodermal lineage marker was observed in eleven cases (3/5 ovarian, 5/15 testicular, and 3/6 extragonadal). Absent to low level expression for all endodermal lineage markers was seen in eight cases (5/15 testicular and 3/6 extra-gonadal). All ovarian tumors showed intermediate to high-level expression of at least one endodermal lineage marker.

High-level TTF-1 staining was limited to ovarian and extra-gonadal yolk sac tumors (2/5 each). Level and intensity of staining were sufficient to cause diagnostic uncertainty for some tumors. For example, case 5 (Figure 1) was a predominantly glandular ovarian tumor $(85 \%)$ in a 31-year-old female. A panel of immunostains showed co-expression of cytokeratin and TTF-1 (strong nuclear staining in $70 \%$ of cells) raising the possibility of malignant struma ovarii, metastatic pulmonary or thyroid neoplasms, or TTF-1-positive ovarian endometrioid carcinoma. Positive staining for SALL4, AFP, and Glyp-3 plus negative Oct-4 allowed a specific diagnosis of pure ovarian yolk sac tumor. Interestingly, additional staining for the purpose of this study also revealed high-level Hep Par 1 expression (weak-moderate cytoplasmic staining in 90\% of 

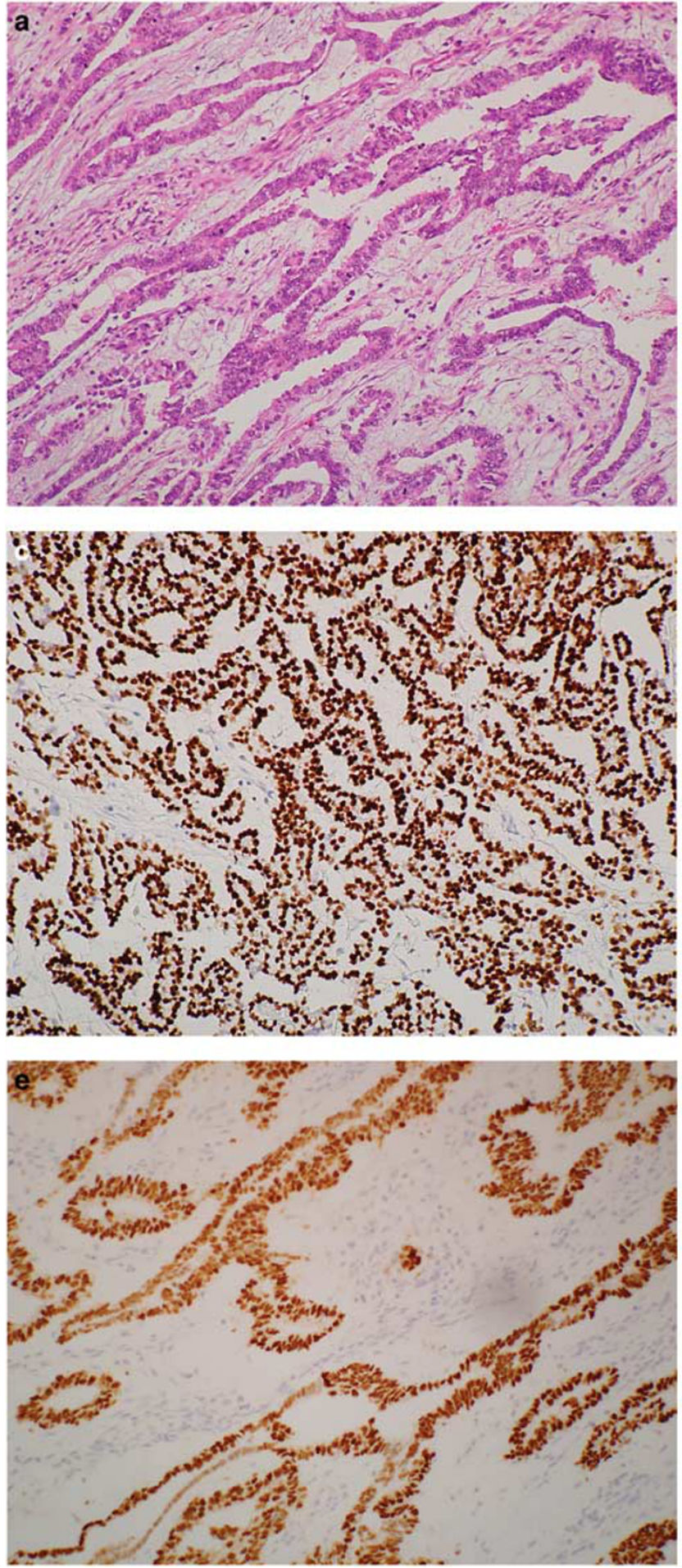
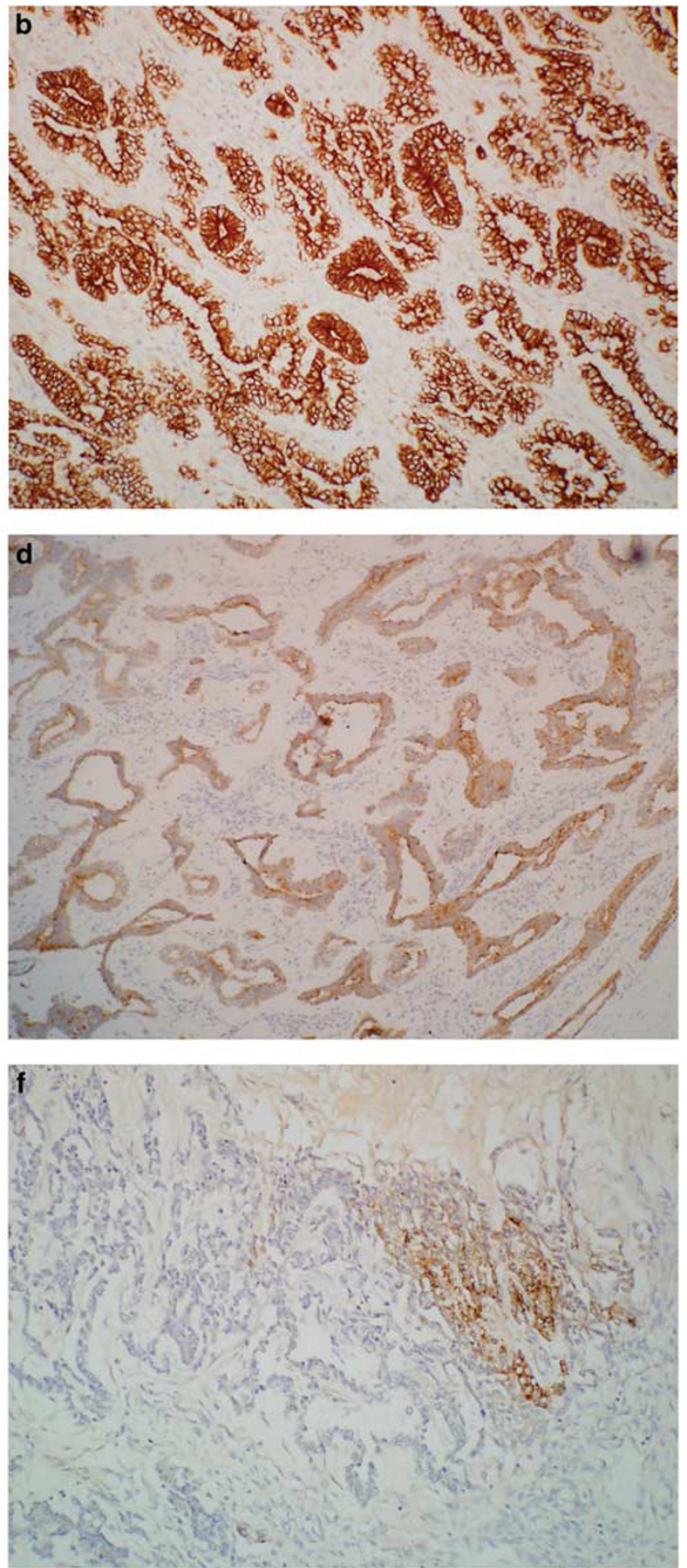

Figure 1 Ovarian yolk sac tumor with endometrioid glandular pattern (case 5). Thirty-one-year-old female with right ovarian mass $(20 \times 16 \times 9 \mathrm{~cm})$ and multiple nodular lesions on peritoneal surfaces of liver, bladder, and omentum. (a) Elongated tubular glands with moderate to severe nuclear atypia in a loose fibromyxoid stroma (H\&E), (b) diffuse strong cytoplasmic staining for CAM 5.2 low molecular weight cytokeratins, (c) diffuse strong nuclear staining for TTF-1, (d) weak-moderate diffuse apical cytoplasmic staining for Hep Par 1, (e) diffuse strong nuclear staining for SALL4, and (f) patchy moderate-strong cytoplasmic staining for AFP (×200, all images).

cells) in this case. A second example (case 22) was a poorly differentiated epithelioid neoplasm within the nasal cavity of a 9-month-old male infant (Figure 2). Diagnosis at intraoperative consultation was poorly differentiated malignancy favor rhabdomyosarcoma. Given the rarity of carcinomas in children, epithelioid features on permanent sections led to staining for AFP and a diagnosis of extra- 

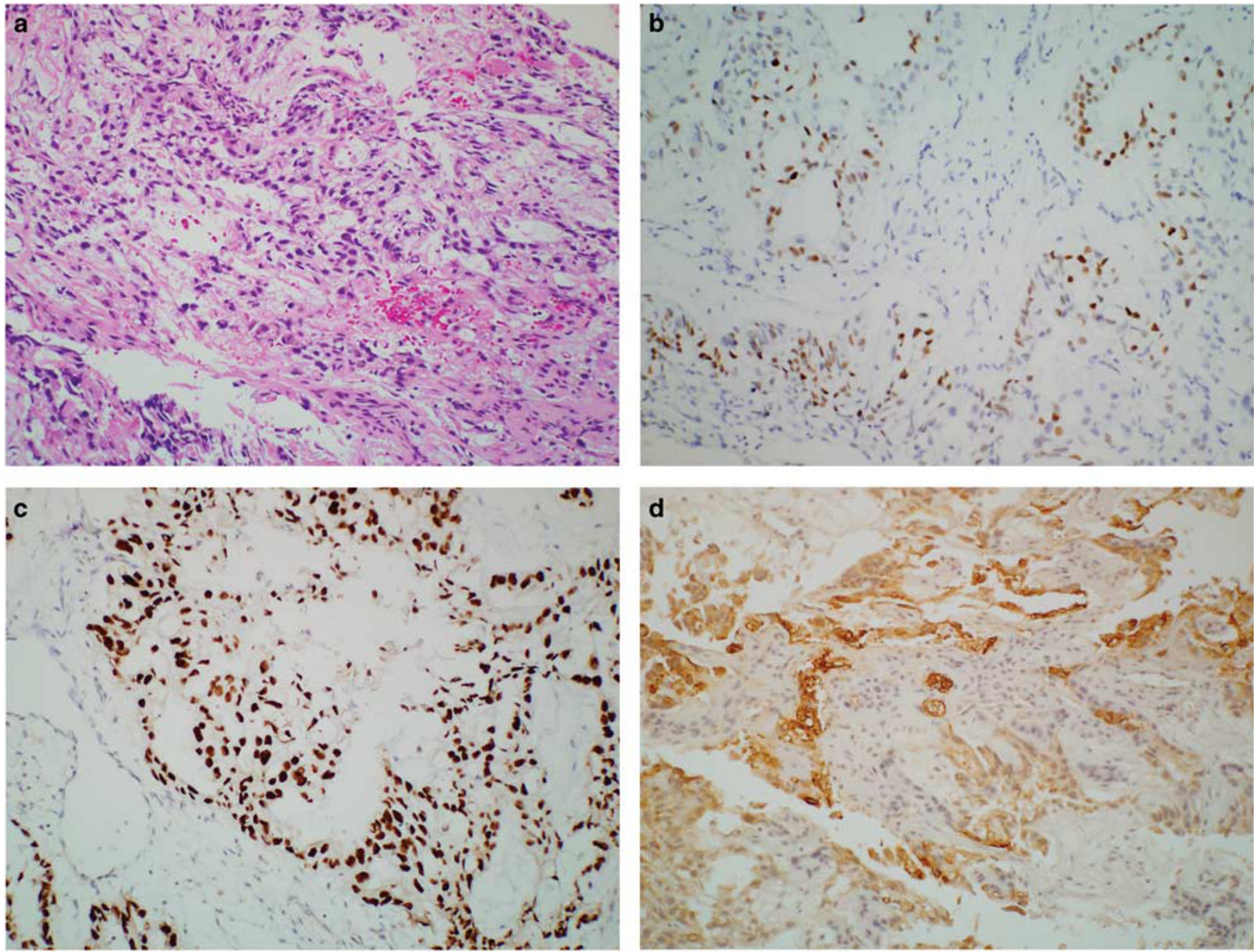

Figure 2 Extra-gonadal yolk sac tumor with mixed reticular/cystic and solid/myxoid features (case 22). Nine-month-old male presented with nasal mass. (a) Microcystic pattern with marked nuclear hyperchromasia and cytoplasmic vacuolation (H\&E), (b) patchy, moderatestrong nuclear positivity for TTF-1, (c) strong diffuse nuclear staining for SALL4, and (d) patchy weak-moderate cytoplasmic staining of microcystic and solid elements with AFP (×200, all images).

gonadal yolk sac tumor. However, in a different scenario, the $25 \%$ positivity for TTF-1 found in this study could have led to consideration of a pituitary, thyroid, or pulmonary neoplasm.

High-level Hep Par 1 staining was never seen in pre-pubertal yolk sac tumors (0/6), but was relatively frequent in older patients $(8 / 20)$. Of note, none of the yolk sac tumors in this study showed foci of overt hepatoid differentiation. High-level CDX2 staining was the least common pattern, observed in only one tumor from each site (adult ovary, adult testis, and pre-pubertal extra-gonadal). Case 17 illustrates the potential pitfalls of aberrant Hep Par 1 and CDX2 staining (Figure 3). This patient presented with a lymph-node metastasis in the neck region following a prior diagnosis of mixed malignant germ cell tumor of testis. Immunostaining showed highlevel positivity (80\%) for both Hep Par 1 (strong cytoplasmic) and CDX2 (weak-moderate nuclear) in reticular/cystic, solid, and glandular areas. Without the clinical history, metastasis from hepatic or gastrointestinal primaries would need to be considered. The correct diagnosis was confirmed by strong co-expression of SALL4 and AFP and negativity for Oct-4.

High-level expression of more than one divergent endodermal lineage marker was seen in four cases. No case showed high-level positivity for all three markers. Two cases co-expressed high-level TTF-1 and Hep Par 1 (both ovarian yolk sac tumor, cases 2 and 5). One case co-expressed high-level TTF-1 and CDX2 (pre-pubertal extra-gonadal yolk sac tumor, case 24) and one case co-expressed high-level CDX2 and Hep Par 1 (lymph node with metastatic testicular mixed malignant germ cell tumor, case 17).

Finally, no invariant associations of divergent endodermal lineage marker expression with histologic subtype were observed. High-level Hep Par 1 staining was predominantly seen in solid and glandular areas, but was occasionally present in reticular/cystic foci (cases 2 and 17). TTF-1 and CDX2 never showed high-level positivity in 

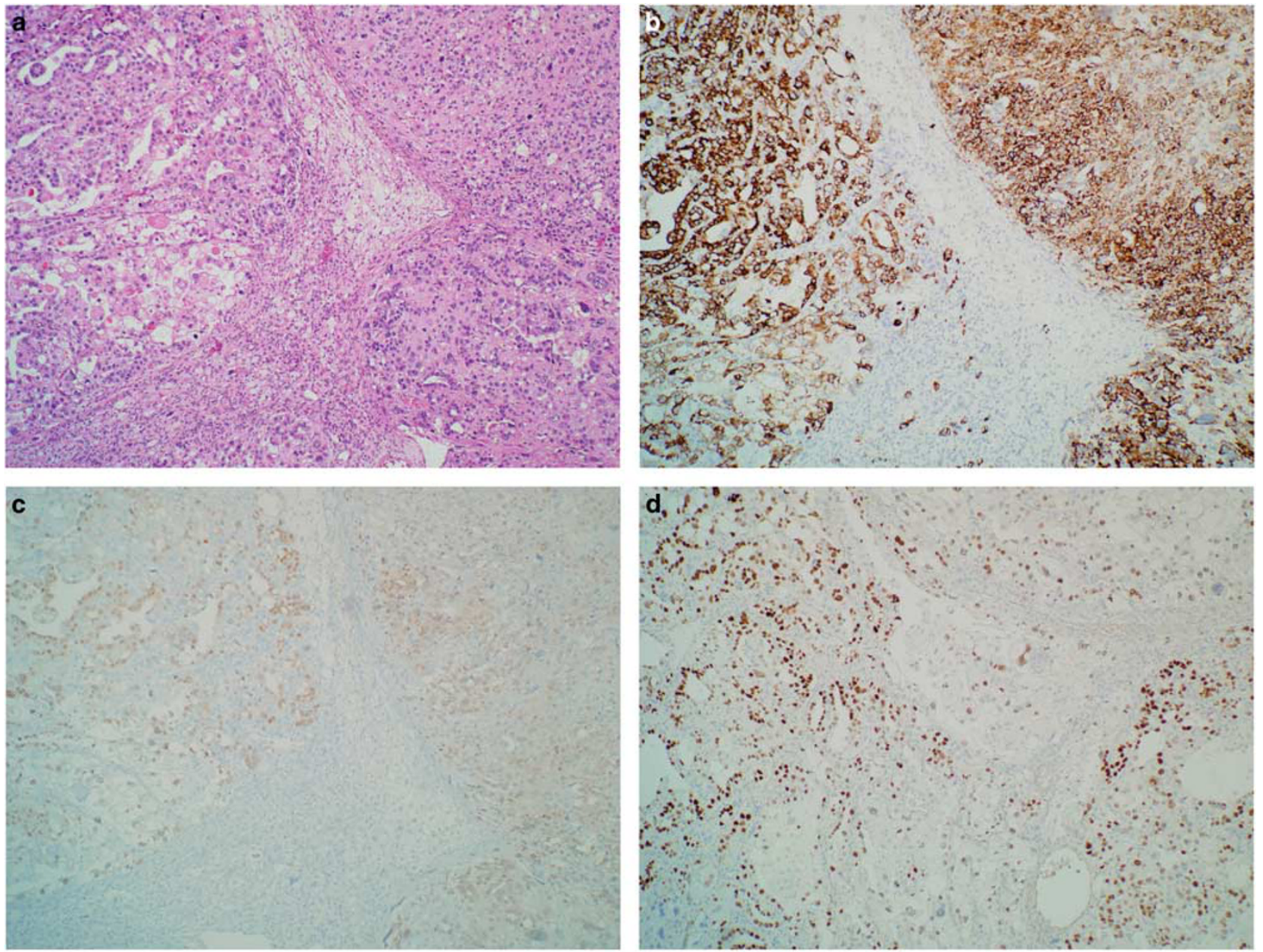

e

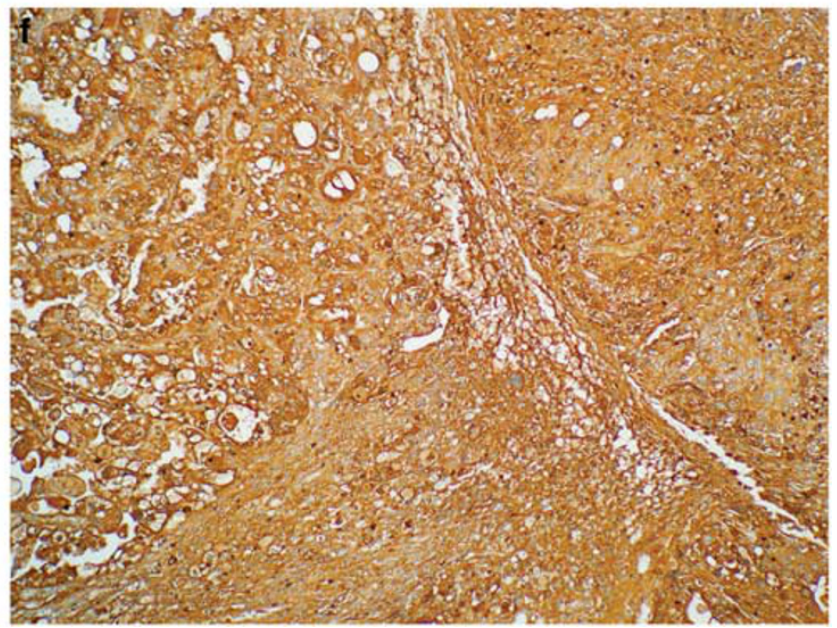

Figure 3 Metastatic mixed malignant germ cell tumor of testis, predominantly yolk sac tumor with cystic, solid, and glandular features (case 17). Thirty-six-year-old male presented with an enlarged lymph node in the left neck. (a) Varied morphology: classic reticular/cystic pattern (left), glandular pattern (bottom right), and solid pattern (top right) (H\&E), (b) diffuse strong cytoplasmic positivity for Hep Par 1, (c) patchy weak-moderate nuclear staining with CDX2, (d) diffuse moderate-strong nuclear staining with SALL4, (e) complete absence of staining with Oct-4, and (f) diffuse strong cytoplasmic staining with AFP (×100, all images).

solid/myxoid foci. Both were most frequent in reticular/cystic and glandular areas, with the latter being most problematic from a diagnostic standpoint as illustrated by case 5 .

\section{Discussion}

The yolk sac is an extra-embryonic structure that has an early role in embryonic nutrition. In some species, 
it has a major role in the function of the placenta. In fact, an older term for yolk sac tumor, endodermal sinus tumor, refers to similarities between yolk sac tumor and the yolk sac placenta of rodents. ${ }^{16-20}$ In humans, the yolk sac becomes a vestigial structure after the first trimester sometimes observed on the placental surface. In rare cases, it can give rise to primary yolk sac tumor of the placenta. ${ }^{21,22}$

Primordial germ cells retain the capacity to differentiate along both embryonic and extraembryonic lines. They can give rise to germ celltype neoplasms (embryonal carcinomas, seminoma in testis, and dysgerminoma in ovary), embryonictype neoplasms (immature and mature teratomas in gonadal or extra-gonadal sites), or extra-embryonictype neoplasms. Extra-embryonic trophoblast stem cell differentiation leads to choriocarcinoma, most commonly in the testis, ${ }^{23}$ while extra-embryonic endodermal stem cell differentiation leads to yolk sac tumor. ${ }^{24-30}$ The regulation of extra-embryonic endodermal stem cells is only partially understood and involves newly recognized transcription factors, such as GATA-4, GATA-6, SOX 7, and SOX 17. ${ }^{31-35}$ Importantly, extra-embryonic endodermal stem cells have the potential to differentiate along endodermal-like lineages and express mature markers associated with thyroid, lung, liver, and gastrointestinal tract. ${ }^{36,37}$

A number of previous studies have addressed divergent expression of endodermal lineage markers in germ cell tumors, mostly in the testis. Early reports documented that both CDX2 and Hep Par 1 could be expressed in a minority of testicular yolk sac tumors. ${ }^{38,39}$ A subsequent study also found occasional CDX2 expression in other malignant germ cell components including choriocarcinoma, teratoma, embryonal carcinoma, and rarely seminoma. ${ }^{40}$ More recent reports have highlighted the differential diagnostic problems arising when CDX2 and/or Hep Par 1 are expressed in somatic-type and glandular forms of yolk sac tumors. ${ }^{41-43}$ Expression of TTF-1 in yolk sac tumor has not been widely studied. One study found no expression in 45 somatic-type yolk sac tumors while a recent review mentions occasional expression in ovarian yolk sac tumors. ${ }^{7,41}$

In this study, yolk sac tumor elements were identified and mapped within gonadal- and extragonadal germ cell tumors occurring in children and young adults. Diagnosis relied on typical histologic patterns and was confirmed with immunohistochemical markers that are sensitive (Glyp-3 and SALL4) and specific (AFP) for yolk sac tumor. Mapping of the exact extent of yolk sac differentiation in mixed malignant germ cell tumors relied on the fact that Oct-4 expression is present in embryonic lineages, but absent in extra-embryonic lineages within yolk sac tumors. Similar to other studies, ${ }^{7,44,45}$ we found that yolk sac tumors were uniformly $100 \%$ positive for SALL4 (high sensitivity) while expression levels for more specific yolk sac tumor markers such as AFP and Glyp-3 were more variable.

TTF-1 is a nuclear protein that regulates transcriptional activity related to organ development and is normally expressed in the adult thyroid, lung, and neurohypophysis. Positive nuclear staining is widely used in the identification of thyroid and pulmonary neoplasms. ${ }^{46,47}$ Importantly, endometrioid-type carcinomas occasionally aberrantly express TTF-1. ${ }^{48,49}$ In this study, we found that a significant number of ovarian and extra-gonadal yolk sac tumors, often with glandular differentiation, strongly expressed TTF-1 in up to $70 \%$ of tumor cells. Testicular yolk sac tumors, pure or mixed, never expressed TTF-1 staining in $>15 \%$ of tumor cells. TTF- 1 staining in ovarian yolk sac tumor with endometrioid-type glandular differentiation is in our opinion the most important pitfall illustrated by this study as exemplified by case 5 in Figure 1.

CDX2 encodes a nuclear transcription factor critical for intestinal embryonic development and is a relatively specific marker of gastrointestinal epithelial tumors (colon, pancreas, stomach). ${ }^{38,50,51}$ It is also expressed in some ovarian mucinous neoplasms. ${ }^{5,53}$ We found high-level positive staining for CDX2 in three cases, one each from adult ovary and testis and an infant with yolk sac tumor arising in a sacrococcygeal teratoma. Staining tended to be weak to moderate in intensity and restricted to reticular/cystic or glandular regions. However, positivity in glandular foci, especially at unusual sites or in metastatic lesions, could present diagnostic problems in selected patients.

Hep Par 1 recognizes a mitochondrial antigen expressed in mature hepatocytes. It is highly sensitive and relatively specific for tumors of hepatocellular origin. Hep Par 1 is strongly and diffusely expressed in hepatoid yolk sac tumors, where it can help confirm the diagnosis. ${ }^{54,55}$ Despite the absence of frankly hepatoid elements in our cases, Hep Par 1 was the most common endodermal lineage marker showing high expression in yolk sac tumors (8 cases). Intermediate to high-level positive staining for Hep Par 1 was found only in tumors from teenagers and adults. Staining was predominantly restricted to myxoid/solid and glandular areas and usually weak-moderate in intensity. As with CDX2, positivity in glandular tumors could occasionally be problematic in tumors at metastatic sites.

Napsin A is involved in the post-translational processing of surfactant protein $B$ and is found primarily in lung and kidney. It is also expressed in mullerian tumors of clear cell type. ${ }^{56,57}$ It is a useful marker distinguishing pulmonary adenocarcinoma (positive) from squamous cell carcinoma (negative) and can help identify adenocarcinomas of unknown origin as originating in lung. ${ }^{58,59}$ Expression of Napsin A in kidney and mullerian clear cell tumors demonstrates that it is not restricted to tissue of endodermal lineage and we did not find any examples of high-level expression in our study. In 
selected instances, a pattern of TTF-1 positivity and Napsin A negativity could be useful for distinguishing yolk sac tumor from a pulmonary neoplasm.

Yolk sac neoplasms are usually easily recognized by virtue of their unique histology and clinical presentation (young patients with gonadal and extra-gonadal germ cell tumors accompanied by elevated serum AFP levels). However, occurrence in much older patients is a recognized phenomenon. ${ }^{60}$ Several situations are well documented in the literature to be problematic: (a) identification of small foci of yolk sac tumor within otherwise benign extra-gonadal teratomas, usually in infancy. In these cases, AFP and Glyp-3 can highlight the areas of yolk sac tumor differentiation, (b) ambiguous epithelioid foci within mixed germ cell tumors of the testis. In these cases, positivity for SALL4, Glyp-3, and/or AFP combined with negative staining for Oct4, CD30, beta hCG (human chorionic gonadotropin), and Inhibin can distinguish yolk sac tumor from potential mimics such as immature teratoma, embryonal carcinoma, and choriocarcinoma, and (c) polyvesicular vitelline yolk sac tumor, particularly in the ovary, which can be negative for AFP. In these cases, Glyp-3 and SALL4 can help to make the diagnosis. ${ }^{10}$

To these situations, we would add: (d) pure ovarian epithelial yolk sac tumors with an endometrioid glandular pattern in adults with positive cytokeratin and TTF-1 staining, which may be mistaken for endometrioid carcinoma, malignant struma ovarii or metastasis from pulmonary or thyroid neoplasms, (e) pure yolk sac tumors in children and adults arising at unusual sites (eg, nasal cavity; case 22), where high-level TTF-1 could lead one to consider spread from thyroid, pituitary, or pulmonary neoplasms, and (f) distant lymph-node metastases in males with occult primary gonadal germ cell tumors where the yolk sac tumor component may aberrantly express CDX2 and/or Hep Par 1, raising the possibility of metastatic gastrointestinal or hepatocellular neoplasms. Extra-gonadal yolk sac tumors in older patients have also been reported to express CDX2 in some cases. ${ }^{44}$

Our study was limited by its retrospective nature and the small number of cases in any one category. Future studies focusing specifically on ovarian tumors with purely glandular morphology and small biopsies with predominantly solid patterns from extra-gonadal sites may provide additional insights. The keys to correct diagnosis in these and other situations are a complete clinical history, consideration of malignant germ cell tumor in the differential diagnosis of all tumors of children and young adults, inclusion of SALL4 in immunohistochemical panels used to interrogate tumors of problematic lineage, knowledge of the many histopathologic variants of yolk sac tumor, and cognizance of the possible range of divergent endodermal lineage marker expression as demonstrated in this study.

\section{Acknowledgments}

We thank Amad Awadallah and the staff of the University Hospitals Case Medical Center Immunohistochemistry laboratory for technical assistance.

\section{Disclosure/conflict of interest}

The authors declare no conflict of interest.

\section{References}

1 Looijenga LH, Oosterhuis JW. Pathogenesis of testicular germ cell tumours. Rev Reprod 1999;4:90-100.

2 Oosterhuis JW, Looijenga LH. Current views on the pathogenesis of testicular germ cell tumours and perspectives for future research: highlights of the 5th Copenhagen Workshop on Carcinoma in situ and Cancer of the TestisAPMIS 2003;111:280-289.

3 Slowikowska-Hilczer J, Romer TE, Kula K. Neoplastic potential of germ cells in relation to disturbances of gonadal organogenesis and changes in karyotype. J Androl 2003;24:270-278.

4 Teilum G. Endodermal sinus tumors of the ovary and testis. Comparative morphogenesis of the so-called mesoephroma ovarii (Schiller) and extraembryonic (yolk sac-allantoic) structures of the rat's placenta. Cancer 1959;12:1092-1105.

5 Ulbright TM, Roth LM, Brodhecker CA. Yolk sac differentiation in germ cell tumors. A morphologic study of 50 cases with emphasis on hepatic, enteric, and parietal yolk sac features. Am J Surg Pathol 1986;10:151-164.

6 Grady RW, Ross JH, Kay R. Patterns of metastatic spread in prepubertal yolk sac tumor of the testis. J Urol 1995;153:1259-1261.

7 Nogales FF, Preda O, Nicolae A. Yolk sac tumours revisited. A review of their many faces and names. Histopathology 2012;60:1023-1033.

8 Pierce GB, Bullock WK, Huntington RW Jr. Yolk sac tumors of the testis. Cancer 1970;25:644-658.

9 Selvaggi SM. Tumors of the ovary, maldeveloped gonads, fallopian tube, and broad ligament. Arch Pathol Lab Med 2000;124:477.

10 Kao CS, Idrees MT, Young RH et al. Solid pattern yolk sac tumor: a morphologic and immunohistochemical study of 52 cases. Am J Surg Pathol 2012;36:360-367.

11 Teilum G. Classification of ovarian tumours. Acta Obstet Gynecol Scand 1952;31:292-312.

12 Teilum G. Classification of testicular and ovarian androblastoma and Sertoli cell tumors; a survey of comparative studies with consideration of histogenesis, endocrinology, and embryological theories. Cancer 1958;11:769-782.

13 Teilum G. Classification of endodermal sinus tumour (mesoblatoma vitellinum) and so-called "embryonal carcinoma" of the ovary. Acta Pathol Microbiol Scand 1965;64:407-429.

14 Teilum G. Classification and interpretation of testicular tumors. Acta Unio Int Contra Cancrum 1953;9:872-874.

15 Ulbright TM. Germ cell tumors of the gonads: a selective review emphasizing problems in differential diagnosis, newly appreciated, and controversial issues. Mod Pathol 2005;18(Suppl 2):S61-S79. 
16 Enders AC, King BF. Formation and differentiation of extraembryonic mesoderm in the rhesus monkey. Am J Anat 1988;181:327-340.

17 Enders AC, Schlafke S, Hendrickx AG. Differentiation of the embryonic disc, amnion, and yolk sac in the rhesus monkey. Am J Anat 1986;177:161-185.

18 Luckett WP. Origin and differentiation of the yolk sac and extraembryonic mesoderm in presomite human and rhesus monkey embryos. Am J Anat 1978;152:59-97.

19 Pierce GB Jr, Midgley AR Jr, Ram JS et al. Pariental yolk sac carcinoma: clue to the histogenesis of Riechert's membrane of the mouse embryo. Am J Pathol 1962;41: 549-566.

20 Stevens LC. Origin of testicular teratomas from primordial germ cells in mice. J Natl Cancer Inst 1967;38: 549-552.

21 Drut R, Mortera M, Drut RM. Yolk sac tumor of the placenta in Wiedemann-Beckwith syndrome. Pediatr Dev Pathol 1998;1:534-537.

22 Matsuyama TA, Kushima M, Yamochi-Onizuka T et al. Placental yolk sac tumor with divergent endodermal differentiation. Int J Gynecol Pathol 2004;23: 398-402.

23 Garcia-Galvis OF, Cabrera-Ozoria C, Fernandez JA et al. Malignant Mullerian mixed tumor of the ovary associated with yolk sac tumor, neuroepithelial and trophoblastic differentiation (teratoid carcinosarcoma). Int J Gynecol Pathol 2008;27:515-520.

24 Garcia RL, Ghali VS. Gastric choriocarcinoma and yolk sac tumor in a man: observations about its possible origin. Hum Pathol 1985;16:955-958.

25 Kawahara M, Takada A, Tachibana et al. Germ cell tumor of the colon with an adenocarcinomatous component. Int J Clin Oncol 2009;14:537-540.

26 Nogales FF, Bergeron C, Carvia RE et al. Ovarian endometrioid tumors with yolk sac tumor component, an unusual form of ovarian neoplasm. Analysis of six cases. Am J Surg Pathol 1996;20:1056-1066.

27 Rutgers JL, Young RH, Scully RE. Ovarian yolk sac tumor arising from an endometrioid carcinoma. Hum Pathol 1987;18:1296-1299.

28 Salem F, Rosenblum MK, Jhanwar SC et al. Teratocarcinosarcoma of the nasal cavity and paranasal sinuses: report of 3 cases with assessment for chromosome 12p status. Hum Pathol 2008;39:605-609.

29 Taylor G, Jordan M, Churchill B et al. Yolk sac tumor of the bladder. J Urol 1983;129:591-594.

30 Thomas J, Adegboyega P, Iloabachie K et al. Sinonasal teratocarcinosarcoma with yolk sac elements: a neoplasm of somatic or germ cell origin? Ann Diagn Pathol 2011;15:135-139.

31 Artus J, Piliszek A, Hadjantonakis AK. The primitive endoderm lineage of the mouse blastocyolk sac tumor: sequential transcription factor activation and regulation of differentiation by Sox17. Dev Biol 2011;350: 393-404.

32 Brown K, Legros S, Artus J et al. A comparative analysis of extra-embryonic endoderm cell lines. PLoS One 2010;5:e12016.

33 Morrisey EE, Tang Z, Sigrist K et al. GATA6 regulates HNF4 and is required for differentiation of visceral endoderm in the mouse embryo. Genes Dev 1998;12: 3579-3590.

34 Schrode N, Saiz N, Di Talia S et al. GATA6 levels modulate primitive endoderm cell fate choice and timing in the mouse blastocyolk sac tumor. Dev Cell $2014 ; 29: 454-467$.
35 Shimoda M, Kanai-Azuma M, Hara K et al. Sox17 plays a substantial role in late-stage differentiation of the extraembryonic endoderm in vitro. J Cell Sci 2007;120: 3859-3869.

36 Marin-Padilla M. Origin, nature and significance of the "embryoids" of human teratomas. Virchows Arch Pathol Anat Physiol Klin Med 1965;340:105-121.

37 Suzuki S, Terauchi M, Umezu T et al. Identification and characterization of cancer stem cells in ovarian yolk sac tumors. Cancer Sci 2010;101:2179-2185.

38 Bing Z, Pasha T, Tomaszewski JE et al. CDX2 expression in yolk sac component of testicular germ cell tumors. Int J Surg Pathol 2009;17:373-377.

39 Fan Z, van de Rijn M, Montgomery K et al. Hep par 1 antibody stain for the differential diagnosis of hepatocellular carcinoma: 676 tumors tested using tissue microarrays and conventional tissue sections. Mod Pathol 2003;16:137-144.

40 Lee MJ, Vogt AP, Hsiao W et al. CDX-2 expression in malignant germ cell tumors of the testis, itratubular germ cell neoplasia, and normal seminiferous tubules. Tumour Biol 2012;33:2185-2188.

41 Magers MJ, Kao CS, Cole CD et al. "Somatic-type" malignancies arising from testicular germ cell tumors: a clinicopathologic study of 124 cases with emphasis on glandular tumors supporting frequent yolk sac tumor origin. Am J Surg Pathol 2014;38:1396-1409.

42 Nogales FF, Quinonez E, Lopez-Marin L et al. A diagnositic immunohistochemical panel for yolk sac (primitive endodermal) tumours based on an immunohistochemical comparison with the human yolk sac. Histopathology 2014;65:51-59.

43 Gonzalez-Roibon N, Katz B, Chaux A et al. Immunohistochemical expression of SALL4 in hepatocellular carcinoma, a potential pitfall in the differential diagnosis of yolk sac tumors. Hum Pathol 2013;44:1293-1299.

44 Cao D, Guo S, Allan RW et al. SALL4 is a novel sensitive and specific marker of ovarian primitive germ cell tumors and is particularly useful in distinguishing yolk sac tumor from clear cell carcinoma. Am J Surg Pathol 2009;33:894-904.

45 Cao D, Humphrey PA, Allan RW. SALL4 is a novel sensitive and specific marker for metastatic germ cell tumors, with particular utility in detection of metastatic yolk sac tumors. Cancer 2009;115:2640-2651.

46 Lau SK, Luthringer DJ, Eisen RN. Thyroid transcription factor-1: a review. Appl Immunohistochem Mol Morphol 2002;10:97-102.

47 Robens J, Goldstein L, Gown AM et al. Thyroid transcription factor-1 expression in breast carcinomas. Am J Surg Pathol 2010;34:1881-1885.

48 Siami K, McCluggage WG, Ordonez NG et al. Thyroid transcription factor-1 expression in endometrial and endocervical adenocarcinomas. Am J Surg Pathol 2007;31:1759-1763.

49 Zhang PJ, Gao HG, Pasha TL et al. TTF-1 expression in ovarian and uterine epithelial neoplasia and its potential significance, an immunohistochemical assessment with multiple monoclonal antibodies and different secondary detection syolk sac tumorems. Int J Gynecol Pathol 2009;28:10-18.

50 Beck F, Stringer EJ. The role of Cdx genes in the gut and in axial development. Biochem Soc Trans 2010;38: 353-357.

51 Silberg DG, Swain GP, Suh ER et al. Cdx1 and cdx2 expression during intestinal development. Gastroenterology 2000;119:961-971. 
52 Vang R, Gown AM, Wu LS et al. Immunohistochemical expression of CDX2 in primary ovarian mucinous tumors and metastatic mucinous carcinomas involving the ovary: comparison with CK20 and correlation with coordinate expression of CK7. Mod Pathol 2006;19:1421-1428.

53 Vang R, Gown AM, Zhao C et al. Ovarian mucinous tumors associated with mature cyolk sac tumoric teratomas: morphologic and immunohistochemical analysis identifies a subset of potential teratomatous origin that shares features of lower gastrointestinal tract mucinous tumors more commonly encountered as secondary tumors in the ovary. Am J Surg Pathol 2007;31:854-869.

54 Butler SL, Dong H, Cardona D et al. The antigen for Hep Par 1 antibody is the urea cycle enzyme carbamoyl phosphate synthetase 1. Lab Invest 2008;88:78-88.

55 Pitman MB, Triratanachat S, Young RH et al. Hepatocyte paraffin 1 antibody does not distinguish primary ovarian tumors with hepatoid differentiation from metastatic hepatocellular carcinoma. Int J Gynecol Pathol 2004;23:58-64.
56 Iwamoto $\mathrm{M}$, Nakatani $\mathrm{Y}$, Fugo $\mathrm{K}$ et al. Napsin $\mathrm{A}$ is frequently expressed in clear cell carcinoma of the ovary and endometrium. Hum Pathol 2015;46:957-962.

57 Yamashita Y, Nagasaka T, Naiki-Ito A et al. Napsin A is a specific marker for ovarian clear cell adenocarcinoma. Mod Pathol 2015;28:111-117.

58 Agackiran Y, Ozcan A, Akyurek N et al. Desmoglein-3 and Napsin A double stain, a useful immunohistochemical marker for differentiation of lung squamous cell carcinoma and adenocarcinoma from other subtypes. Appl Immunohistochem Mol Morphol 2012;20:350-355.

59 Whithaus K, Fukuoka J, Prihoda TJ et al. Evaluation of napsin A, cytokeratin 5/6, p63, and thyroid transcription factor 1 in adenocarcinoma versus squamous cell carcinoma of the lung. Arch Pathol Lab Med 2012;136: 155-162.

60 Roma AA, Przybycin CG. Yolk sac tumor in postmenopausal patients: pure or associated with adenocarcinoma, a rare phenomenon. Int J Gynecol Pathol 2014;33:477-482. 